\title{
Memory, attention and fluency deficits in COPD may be a specific form of cognitive impairment
}

\author{
Charlotte Morris ${ }^{1,6}$, James W. Mitchell ${ }^{1,6}$, Hannah Moorey ${ }^{1}$, \\ Helen-Cara Younan ${ }^{2}$, George Tadros ${ }^{3,4}$ and Alice M. Turner $\mathbb{C}^{1,5}$
}

Affiliations: ${ }^{1}$ University Hospitals Birmingham Foundation Trust, Birmingham, UK. ${ }^{2}$ Faculty of Medicine, Imperial College, London, UK. ${ }^{3}$ Aston Medical School, Birmingham, UK. ${ }^{4}$ Birmingham and Solihull Mental Health NHS Foundation Trust, Birmingham, UK. ${ }^{5}$ University of Birmingham, Birmingham, UK. ${ }^{6}$ These authors contributed equally.

Correspondence: Charlotte Morris, Bryntirion House, Cookes Lanes, Rudheath, Cheshire, CW9 7RS, UK. E-mail: charlotte.morris4anhs.net

ABSTRACT There is increasing evidence demonstrating an association between chronic obstructive pulmonary disease (COPD) and cognitive impairment. We present a narrative review of published studies on the subject and a cross-sectional study investigating domain-specific cognitive impairment in people with COPD compared to people with known Alzheimer's dementia, and controls without known COPD or cognitive impairment. The aim of the study was to compare prevalence and pattern of cognitive impairment between the three groups using the Addenbrooke's Cognitive Examination (ACE)-III tool.

A total of 89 participants were recruited (44 with COPD, 17 with Alzheimer's and 28 controls).

Patients with COPD had significantly lower total ACE-III scores than controls $(\mathrm{p}<0.001)$. When comparing the COPD group to the known Alzheimer's dementia group, overall ACE-III scores were significantly lower in the Alzheimer's dementia group than the COPD group $(p=0.019)$. The domainspecific scores for attention $(p<0.004)$, memory $(p<0.004)$ and fluency $(p<0.001)$ were significantly lower in the Alzheimer's dementia group than the COPD group.

Our result suggest that the COPD group were significantly more likely to have cognitive impairment than the healthy control group. This was supported by the results of a narrative review of the published literature. Our results show that the pattern of impairment in the COPD group is different to the pattern of impairment shown in the known Alzheimer's dementia group, with significant differences in the cognitive domains affected. These results are in keeping with the findings of other previously published studies included in the narrative review.

$@$ ERSpublications

People with COPD are more likely than controls to have cognitive impairment. When compared to people with known Alzheimer's disease, the pattern of impairment and cognitive domains affected are significantly different. http://bit.ly/2VPFxjh

Cite this article as: Morris C, Mitchell JW, Moorey H, et al. Memory, attention and fluency deficits in COPD may be a specific form of cognitive impairment. ERJ Open Res 2019; 5: 00229-2018 [https://doi.org/10.1183/23120541.00229-2018].

This article has supplementary material available from openres.ersjournals.com

Received: Nov 302018 | Accepted after revision: April 012019

Copyright $\odot$ ERS 2019. This article is open access and distributed under the terms of the Creative Commons Attribution Non-Commercial Licence 4.0. 


\section{Introduction}

The typical profile of patients with chronic obstructive pulmonary disease (COPD) includes a wide range of comorbidity. There is increasing evidence demonstrating an association between COPD and cognitive impairment [1-3]. With an ageing population and the consequent rise in the prevalence of cognitive impairment, this is a topical area of research needing further investigation, particularly in terms of the domains of cognition affected in COPD patients. Prevalence estimates of cognitive impairment in COPD in previously reported studies vary widely, ranging from $2 \%$ to $52 \%$; how this compares to age-matched controls is unclear. A number of suggestions have been made regarding the underlying pathophysiology, including cerebral hypoxia, loss of hippocampal volume and inflammatory mediator-related neuronal damage $[2,4]$.

There is suggestion that people with COPD have different areas of cognitive ability affected than those diagnosed with Alzheimer's dementia or other known types of dementia [1-4]. If confirmed, this would be clinically relevant for COPD management (e.g. it might alter the ability to comply with particular treatment types or benefit from more complex therapies) and for care of their comorbid disease, as specific types of cognitive impairment may have different management strategies [5]. What is unclear is if there is a specific type of neurocognitive dysfunction related to COPD and little literature relates to the direct investigation of this.

This article presents a literature review that examines the peer-reviewed, published literature regarding the link between COPD and cognitive impairment, specifically examining evidence for a discrete type of impairment in those with COPD, and its mediating factors. The narrative conclusions from the review of the literature led to our conducting a cross-sectional study investigating domain-specific cognitive impairment in people with COPD compared to patients with known Alzheimer's-type dementia and healthy controls without known COPD or cognitive impairment, the results of which are also reported here. The aim was to compare the prevalence and pattern of cognitive impairment between the three groups.

\section{Methods}

\section{Literature review}

A comprehensive search was performed of the PubMed database and the Cochrane Database of Systematic Reviews for papers published between January 2010 and January 2015 concerning cognition and COPD. Table 1 summarises inclusion criteria. References from previous systematic reviews prior to 2010 were extracted. Standard methods to eliminate bias were employed. Two reviewers examined retrieved titles and abstracts, and where appropriate, manuscripts. Papers included after the title/abstract stage were read by two authors (H-C. Younan and C. Morris) and reviewed for inclusion. Studies were reviewed to determine "themes" of clinical or mechanistic relevance, with a view to meta-analysis. Since study heterogeneity precluded this, narrative conclusions and comparisons became the focus of our output. Further details on review methodology are detailed in the supplementary material.

\section{Methods for cross-sectional study \\ Participants}

We enrolled patients with moderate to severe COPD from a secondary care respiratory clinic in a medium-sized urban teaching hospital in the UK over an 8-month study period from June 2016 to April 2017. Moderate to severe COPD was defined as patients with a forced expiratory volume in $1 \mathrm{~s}(\mathrm{FEV} 1)$ of $<80 \%$ predicted.

\section{TABLE 1 Criteria for inclusion in the review}

Study designs Clinical trials, epidemiological studies, observational studies, cohort studies, review articles or case-control studies exploring a direct link between COPD and cognitive impairment

Patients Intervention

Comparator Outcomes
Patients diagnosed with COPD

Cognitive testing using various cognitive testing tools, including domain-specific cognitive tools

Patients without known/diagnosed COPD

Cognitive impairment as defined by the specified tools used in the individual studies Domains of cognition affected/domain specific scores on the cognitive testing tools

COPD: chronic obstructive pulmonary disease. 
As a comparator, patients with known cognitive impairment and a formal diagnosis of Alzheimer's dementia but without COPD were recruited from the Join Dementia Research Network. Control patients with a similar age profile and sex distribution but without a diagnosis of COPD or cognitive impairment were also recruited.

All subjects gave their informed, written consent in accordance with the Declaration of Helsinki and following approval from the National Health Service Local Research Ethics Committee (ref. 16/EM/0192)

\section{Assessment of cognition}

We used the Addenbrookes Cognitive Examination (ACE)-III to assess cognitive impairment. The ACE-III is a validated tool that can be used to establish areas of cognition affected in different types of dementias [6] Cut-off scores for possible dementia and mild cognitive impairment are 82 and 88 out of 100, respectively. The ACE-III investigates six different domains of cognition: memory, attention, perception, visuospatial abilities, language and verbal fluency.

In a subgroup, data regarding baseline oxygen saturations, FEV1 and $\mathrm{FEV}_{1} \%$ pred values were available. Data regarding FEV1 and FEV1 \% pred were available for 29 participants (28 in the COPD group and one control). Data for baseline oxygen saturations were available for 28 participants (27 in the COPD group and one control). We completed further analysis comparing overall and domain-specific scores compared to baseline oxygen saturations, FEV1 and FEV1 \% pred.

\section{Statistical analysis}

All statistical analyses were carried out in SPSS version 25 (IBM, Armonk, NY, USA). We analysed the difference in ACE-III total and domain-specific scores between groups. Dunn's pairwise tests were then carried out for two pairs of groups (COPD group compared to cognitive impairment group and COPD group compared to control group) for each analysis. The significance values were then adjusted using Bonferroni corrections, given multiple group comparisons.

ANOVA was then performed to control for cardiovascular disease (CVD) and smoking. A Dunnet's pairwise multicomparison test was then performed to compare the COPD and cognitive impairment group and the COPD and control group. We used linear regression with Pearson's correlation coefficient and Spearman rank correlation to compare the baseline saturations, FEV1 and FEV1 \% pred data to overall and domain-specific ACE-III scores.

\section{Results}

\section{Results of narrative review}

We retrieved 327 original articles of which 18 met the inclusion criteria. A further 94 were retrieved from the reference list of a prior systematic review of which 13 were included; 31 met the inclusion criteria for the final review. Table 2 describes summary characteristics of the included studies and major findings (see the supplementary material for detail and a Preferred Reporting Items for Systematic Reviews and Meta-Analyses diagram).

The included studies did not specify the type of dementia or major neurocognitive disorder present in subjects and rarely specified if dementing conditions with a known pathophysiology (such as alcoholic dementia) were excluded. The 31 included papers employed psychometric tests to establish the level of impairment in cognition and domains affected, but did not translate the results of these cognitive tests into clinical diagnoses. As different studies used different psychometric tests the definition of cognitive impairment was not standardised and differed in the different papers included in the review.

Four papers studied cognitive outcomes according to COPD severity [7-10]. However, one of these papers used "self-reported history of COPD" rather than pulmonary function testing to classify COPD severity [10]. Varying criteria were used to diagnose COPD in the included studies. These variations in definitions and methodological limitations affected the quality of included studies and the inferences that can be made, although it seemed cognitive impairment was increased in COPD patients and largely affected memory.

10 of the studies included in the review discussed effects of hypoxia or hypoxaemia on cognitive impairment, with two concluding that there is a relationship between arterial oxygen tension $\left(\mathrm{PaO}_{2}\right)$ and severity of cognitive impairment $[7,11]$. Reduced $\mathrm{PaO}_{2}$ particularly related to impairment in the areas of motor function, attention and processing speed. However, this association was inconsistent; DoDD et al. [1] found that cognitive function was only mildly impaired in patients without hypoxaemia. ANTONELLI-INCALZI et al. [12] included hypoxic-hypercapnic COPD cases and found that $48.5 \%$ had a form of cognitive deterioration; longer duration of hypercapnia and hypoxia was significantly correlated to cognitive impairment. Other mechanisms were considered by included studies; however, there was relatively little 


\begin{tabular}{|c|c|}
\hline \multicolumn{2}{|l|}{ Number of patients } \\
\hline COPD & 11171 \\
\hline Other forms of cognitive impairment diagnosed prior to inclusion & 46 \\
\hline Controls & 48689 \\
\hline Age years mean & 67.53 \\
\hline \multicolumn{2}{|l|}{ Cognitive impairment instruments used in more than one study } \\
\hline Halstead-Reitan Test Battery & 3 \\
\hline Wechsler Adult Intelligence Scale & 3 \\
\hline Mental Deterioration Battery & 3 \\
\hline Trail Making Test A-D & 7 \\
\hline Wechsler Test of Adult Reading & 2 \\
\hline Mini Mental State Examination & 14 \\
\hline Wechsler Memory Scale-III test battery & 5 \\
\hline Grooved pegboard test & 2 \\
\hline Seashore Rhythm Test & 3 \\
\hline \multicolumn{2}{|l|}{ Study design } \\
\hline Cohort & 7 \\
\hline Case-control & 14 \\
\hline Observational & 2 \\
\hline Cross-sectional & 2 \\
\hline Interventional & 1 \\
\hline Review articles (not included in table) & 5 \\
\hline \multicolumn{2}{|l|}{ Studies reporting COPD and cognitive impairment } \\
\hline An association between COPD and cognitive impairment & 20 \\
\hline \multirow{2}{*}{\multicolumn{2}{|c|}{ Studies with statistically significant differences seen in COPD" }} \\
\hline & \\
\hline Memory & 5 \\
\hline Attention & 3 \\
\hline Fluency & 4 \\
\hline Language & 2 \\
\hline Visuospatial & 3 \\
\hline
\end{tabular}

consistency to the findings (supplementary material). Since the review findings were somewhat limited, we felt validation work was necessary, which comprised the second part of our study.

\section{Cross-sectional study}

A total of 89 participants were recruited over the study period (44 with COPD, 17 with Alzheimer's and 28 controls). Recruitment was challenging, particularly for the known Alzheimer's dementia group, eventually resulting in use of a national network to enhance case ascertainment. Comparison of baseline characteristics demonstrated no significant difference between the three groups except for smoking exposure $>10$ pack-years and cardiovascular comorbidity, which were more frequently observed in the COPD group (table 3).

Patients with COPD had significantly lower total ACE-III scores compared to the healthy controls $(\mathrm{p}<0.001)$. 50\% (95\% CI 33.8-66.2\%) of COPD patients demonstrated scores below the cut-off for cognitive impairment (88 out of 100). This compares to just $7 \%$ (95\% CI 9.0-23.5\%) of healthy control group participants. The distribution of total ACE-III scores is presented in figure 1.

\section{Domain-specific scores}

In patients with COPD, memory and fluency subscores were significantly lower compared to healthy control group participants whilst other cognitive domains remained intact. Whilst patients with COPD had lower total ACE-III scores compared to healthy controls (COPD group median 87.5 (interquartile range (IQR) 84-91) versus control group median 96 (IQR 93-97)) scores were significantly higher than for patients in the known Alzheimer's dementia group (median 79, IQR 70-80).

There were significant differences in the overall ACE-III scores $(p=0.019)$ and in the attention $(p=0.004)$, memory $(\mathrm{p}=0.004)$ and fluency subscores $(\mathrm{p}<0.001)$ between the COPD group and the known Alzheimer's dementia group. Those with COPD scored significantly higher overall and in these domains. 


\section{TABLE 3 Demographic and clinical characteristics}

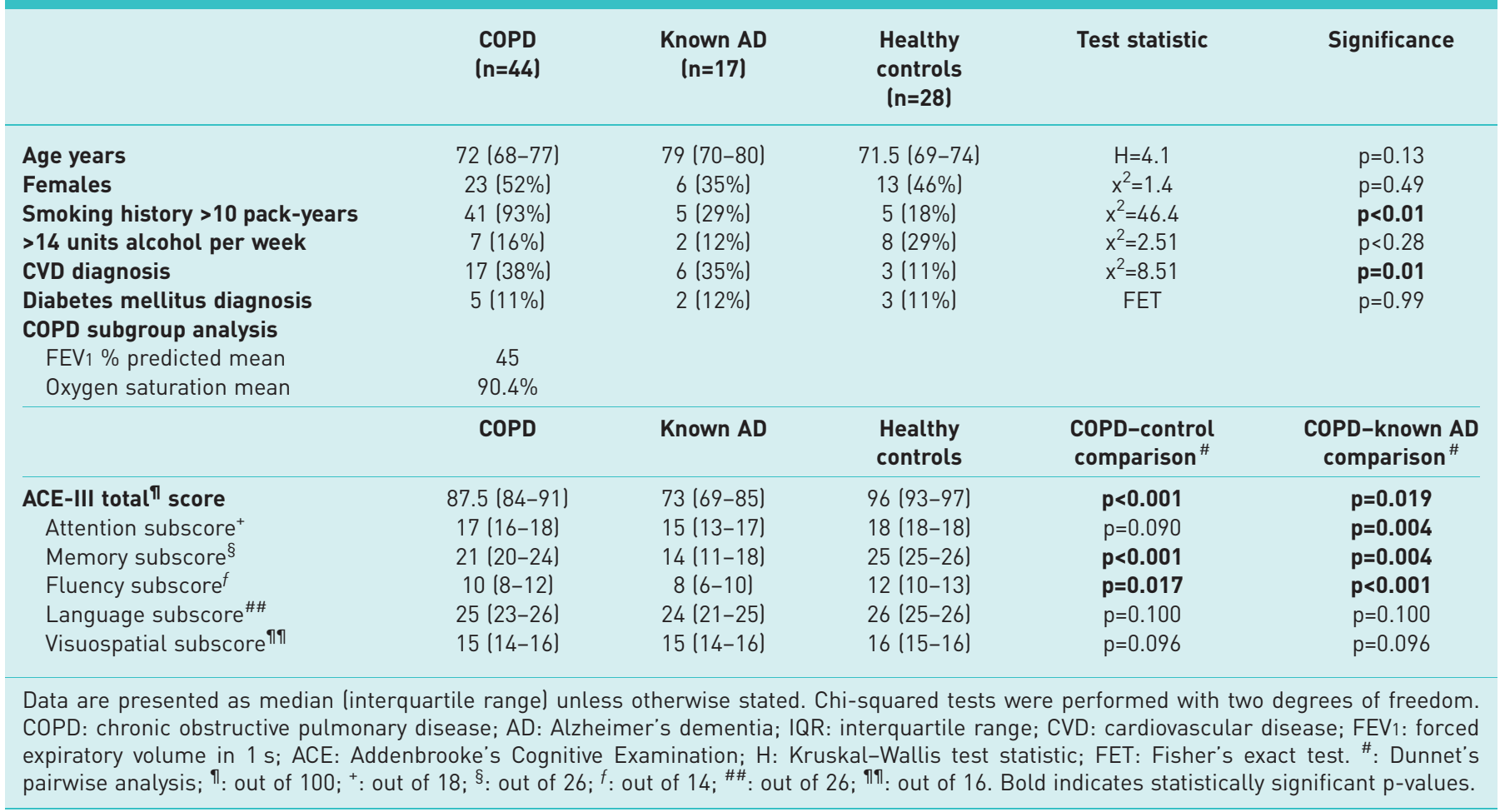

Lower baseline saturations were significantly correlated with lower overall scores in the domain of fluency $(\mathrm{p}<0.01)$. Baseline saturations were not correlated with overall ACE-III score or with any of the other cognitive domains. Lower FEV1 was correlated with lower ACE-III scores in the domains of memory $(\mathrm{p}=0.03)$ and fluency $(\mathrm{p}=0.04)$ but not overall ACE-III score (tables 4-6).

\section{Discussion}

Studies included in the review demonstrated increased prevalence of cognitive impairment in COPD patients compared to controls but the quality and heterogeneity of studies limited conclusions that could be drawn. Particularly important was that diagnosis of cognitive symptoms was rarely precise. It was

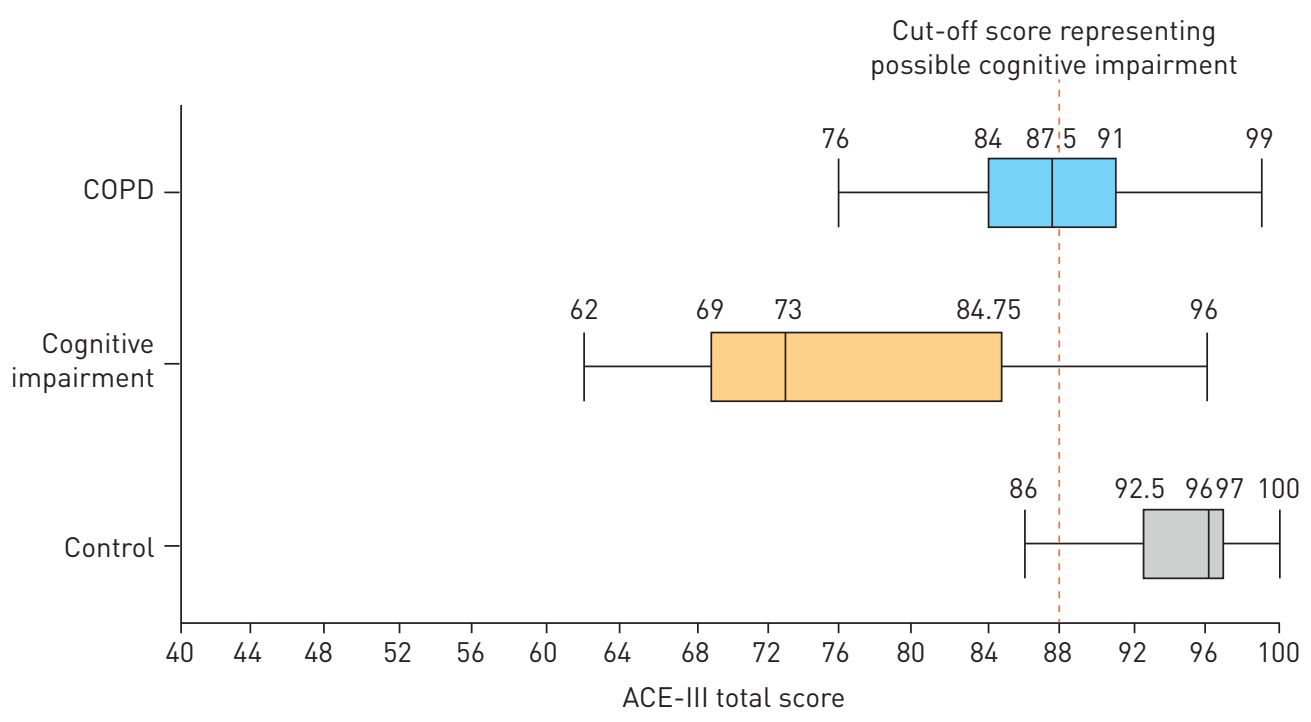

FIGURE 1 Distribution of Addenbrooke's Cognitive Examination (ACE)-III total scores. COPD: chronic obstructive pulmonary disease. 
TABLE 4 Baseline saturations compared to overall Addenbrooke's Cognitive Examination (ACE)-III score and domain-specific ACE-III score ( $n=28$ )

Pearson correlation coefficient p-value for saturations versus overall score and specific domains

$\begin{array}{lcc}\text { Overall ACE-III score } & 0.02 & 0.91 \\ \text { Attention } & 0.11 & 0.58 \\ \text { Memory } & 0.11 & 0.741 \\ \text { Fluency } & -0.49 & \mathbf{0 . 0 0 6 7} \\ \text { Language } & 0.06 & 0.756 \\ \text { Visuospatial } & -0.01 & 0.944\end{array}$

Bold indicates statistically significant $p$-values.

TABLE 5 Forced expiratory volume in $1 \mathrm{~s}$ (FEV1) compared to overall Addenbrooke's Cognitive Examination (ACE)-III score and domain-specific ACE-III score (n=29)

Spearmann's rank correlation coefficient

Overall ACE-III score
Attention
Memory
Fluency
Language
Visuospatial

Bold indicates statistically significant $p$-values. p-value for FEV1 versus overall score and specific domains

0.0651

0.069

0.0262

0.0361

0.2834

0.3583

TABLE 6 Forced expiratory volume in $1 \mathrm{~s}$ (FEV1) \% predicted compared to overall

Addenbrooke's Cognitive Examination (ACE)-III score and domain-specific ACE-III score (n=29)

\begin{tabular}{lcc} 
& $\begin{array}{c}\text { Spearmann's rank correlation } \\
\text { coefficient }\end{array}$ & $\begin{array}{c}\text { p-value for FEV } 1 \text { \% predicted versus overall } \\
\text { score and specific domains }\end{array}$ \\
\hline Overall ACE-III score & -0.109 & 0.282 \\
Attention & -0.1097 & 0.0901 \\
Memory & -0.251 & 0.1782 \\
Fluency & -0.114 & 0.2741 \\
Language & 0.1144 & 0.2736 \\
Visuospatial & -0.162 & 0.196 \\
\hline
\end{tabular}

therefore necessary to conduct a study to determine the pattern of cognitive impairment present in COPD. Dementia is an umbrella term that describes a large number of diseases that have different pathophysiological processes leading to different patterns of cognitive impairment. There are many different types of dementia and cognitive impairment leading to very different clinical pictures; the fifth edition of the Diagnostic and Statistical Manual of Mental Disorders [13] now refers to "major neurocognitive disorder" in an attempt to better define conditions that have cognitive impairment as the primary feature.

In our cross-sectional study, we included patients with known Alzheimer's disease and compared these to people with COPD. There were significant differences in the overall ACE-III scores and attention, memory and fluency subscores between the COPD group and the known Alzheimer's dementia group. These results may suggest that the pattern of impairment in COPD was different, with our results showing that the attention, memory and fluency domains in COPD patients was preserved compared to those with known Alzheimer's dementia. This adds weight to the idea that people with COPD have a different pattern of impairment compared to those with other known causes of cognitive impairment and as such, 
COPD-associated cognitive impairment may represent a new and different type of major neurocognitive disorder. An alternative interpretation is that in our study, those with COPD had less advanced impairment compared to those with known Alzheimer's dementia.

\section{Prevalence of cognitive impairment}

In people aged $>65$ years, dementias occur in approximately $5-8 \%$ and cognitive impairment in different degrees in $5-20 \%$ [14]. Included studies in the review showed a range of $2-52 \%$ of COPD patients to be affected, across age groups, although few studies used this as the specific outcome, choosing in many cases to focus on areas of cognition instead. In our cross-sectional study, the prevalence of cognitive impairment, as defined by the cut-off score in the ACE-III, in the COPD group was 50\% (95\% CI 33.8-66.3\%). This prevalence rate is towards the higher end of those studies included in the review. There is wide variation in the prevalence of cognitive impairment in COPD in the studies included in our review. We hypothesise that the most important, likely reasons for this are the lack of consensus regarding the definition of cognitive impairment and use of different cognitive assessment tools; even studies that used the same cognitive tests had varying thresholds for the definition of cognitive impairment. Most commonly used was the Mini Mental State Examination (MMSE), which was used in 14 studies; it has been suggested that MMSE "should not be used as a diagnostic tool to identify dementia" [15] and may underestimate impairment compared to other cognitive tests $[16,17]$. The MMSE score can be affected by age, education and cultural background [15]; studies did not always control for these factors when interpreting scores. This underestimation of impairment with the MMSE in many of the included studies in the review, coupled with the use of the ACE-III in our cross-sectional study, may have contributed to the prevalence of cognitive impairment we found in COPD patients.

\section{Domains of cognition affected in COPD}

Cognitive function is classified into domains: perception, attention, memory and learning, abstract thinking and executive function, language, and intelligence [18]. Of these, memory was the one most consistently impaired in both the review and our validation study $[1,12,19-21]$, though some studies have reported broader deficits spanning memory, attention, symbolic representation, visual processing and shifting capacity [22]. Neuroimaging studies [21, 23] have found reduced perfusion of frontal and subcortical regions in COPD, possibly explaining executive dysfunction and attention deficits seen $[1,19]$. Fluency was affected in our cross-sectional study; evidence from the review supported this.

From the included studies in the review, it was difficult to determine whether COPD associated with Alzheimer's or vascular dementia, perhaps through a confounding factor such as smoking, or was associated with a specific pattern of cognitive impairment unique to the condition. It is known that different areas of cognition are affected in different dementias; for example, in Alzheimer's dementia, memory, executive function, language, praxis and visuoconstructional abilities are primarily affected [24]. The consistent memory and fluency impairment of COPD seen in the review, and confirmed by our cross-sectional study, may be a specific form of cognitive impairment. Cognitive impairment, particularly in the memory and attention domains, may explain the poor adherence to inhaler therapy and health behaviour change seen in this population [7].

Dementia is a devastating condition in terms of quality of life. COPD patients are increasingly recognised as being at high risk for cognitive impairment and subsequent dementia. Clinically, this is important to allow for appropriate modifications in lifestyle and drug therapies. For example, if praxic skills are impaired in COPD, inhaled therapies would be difficult to self-administer; if verbal memory and attention are impaired, pulmonary rehabilitation may need modifications to bring greater benefit.

\section{Risk factors for cognitive impairment related to COPD}

Patients with COPD have progressive airflow limitation and emphysematous changes in the lungs, resulting in a ventilation-perfusion mismatch, potentially causing chronic hypoxaemia [25]. Alternatively, the association between cognitive impairment and COPD could be explained by altered, decreased cerebral perfusion mediated by vascular disease. There is widespread evidence suggesting hypoxaemia alone does not account for cognitive changes seen in COPD. Studies in both inpatients and outpatients have reported cognitive deterioration even in COPD subjects without severe hypoxaemia. In our cross-sectional study, lower baseline saturations were significantly correlated with lower ACE-III scores in the fluency domain but overall cognitive scores were not affected; as blood gases were not measured in our study, it is difficult to comment on the contribution of hypoxia as a mediating factor in development of cognitive impairment.

FEV1 has been explored in relation to cognitive impairment in COPD. CLEUTJENs et al. [20] found worsening cross-sectional memory and numeric short-term memory were associated with worsening FEV1. However, FroHnhofen et al. [26] found that only $78 \%$ of their cohort and $47 \%$ of those with cognitive impairment 
could adequately complete lung function testing. Therefore, it is difficult to accurately assess any association of cognitive impairment and $\mathrm{FEV}_{1}$ if cognitively impaired patients cannot perform spirometry. In our cohort, lower FEV1 was significantly correlated with lower scores on the ACE-III in the domains of memory and fluency. The mechanism for this, if unrelated to hypoxia, is not clear.

\section{The relevance of comorbidity to cognitive impairment in COPD}

Those with COPD are often smokers or ex-smokers and are older; they are likely to have significant comorbidities [22]. In keeping with this, in the cross-sectional study, baseline differences in comorbidities were observed between the COPD group and the control groups in CVD diagnosis and smoking history $>10$ pack-years. The effect of comorbidities in COPD and cognitive dysfunction was investigated by some of the included studies in the review. DAL Negro et al. [22] did not find any relationship between cardiovascular and metabolic comorbidity and cognitive dysfunction, concluding that comorbidities were unlikely to account for the cognitive dysfunction seen in COPD.

VILLENEuve et al. [19] found that although patients with COPD had more vascular comorbidity, the number and extent of vascular comorbidities was similar between the COPD patients with and without cognitive impairment, implying that vascular comorbidities did not have a significant effect on cognition. In our cross-sectional study, adjustments for smoking and known vascular disease were made and differences in cognitive impairment remained. These adjustments were intended to act as a surrogate for undiagnosed cerebrovascular disease and, whilst imperfect compared to formal diagnosis by neuroimaging, imply that the cognitive impairment in COPD cannot be simply put down to being a cerebrovascular pathological process.

\section{Strengths and limitations}

The review augments prior work as our inclusion criteria allowed a wider overview of the research supporting interaction between COPD and cognitive impairment, highlighting that there are mediators of cognitive impairment that are specific to COPD, and determining key areas for future research. The strengths and limitations of our review are discussed in the supplementary material.

A major strength of the cross-sectional study is use of the ACE-III, which is sensitive and allows investigation of domain-specific cognitive function. Inclusion of known dementia patients was important, as this allowed us to show the pattern of cognitive impairment in COPD was different to that in Alzheimer's disease.

There are a number of limitations that should be considered when interpreting the findings of the study. This pilot study had a small sample size but despite this, shows interesting results suggesting further, larger scale studies into this area are warranted. Patients and controls were not matched on a case by case basis, in order to facilitate recruitment. Despite this, differences between groups were accounted for in the analysis. We did not employ biochemical, neuroimaging or neurophysiological methods to explore the underlying pathogenesis of cognitive impairment in patients with COPD. A larger study including people with other types of dementia (e.g. vascular dementia or Lewy body disease) could further clarify whether the cognitive domains affected for each type of dementia differ from the COPD group, thus giving further evidence supporting a COPD-associated pattern of impairment. Issues with recruitment, particularly in the established cognitively impaired population, precluded this in our centre.

We did not conduct blood gas analyses on patients involved in the study. This is a limitation as hypercapnia and hypoxia were found to be correlated with cognitive impairment in previous studies included in our narrative review. In further, larger studies into this area, it would be useful to conduct blood gas analyses at the time of data collection, and investigate whether there is any association between these and ACE-III scores.

\section{Conclusions}

The cross-sectional study demonstrates a high proportion of cognitive impairment in patients with COPD. This is in keeping with our findings in the literature review. The cognitive domains of attention, memory and fluency seem to be predominantly affected in our cohort. We cannot conclude that there is evidence of a specific, discrete type of cognitive impairment unique to COPD based on the results of this cross-sectional study and literature review alone; however, our findings further our understanding of the pattern of cognitive impairment in patients with COPD and may help explain the poor treatment adherence seen in this population. Further studies should seek to elucidate the underlying neurobiological mechanisms explaining this association.

Acknowledgements: The researchers would like to thank Join Dementia Research for assistance in recruiting patients to this study. Join Dementia Research is funded by the Dept of Health and delivered by the National Institute for Health Research in partnership with Alzheimer Scotland, Alzheimer's Research UK and the Alzheimer's Society (www.joindementiaresearch.nihr.ac.uk). 
Conflict of interest: C. Morris has nothing to disclose. J.W. Mitchell has nothing to disclose. H. Moorey has nothing to disclose. Helen-Cara Younan has nothing to disclose. G. Tadros has nothing to disclose. A.M. Turner reports personal fees for education talks and nonfinancial support for meeting attendance from Boehringer Ingelheim, a research grant in AATD from Grifols Biotherapeutics, and a research grant in airway disease in older people, and fees to attend conferences and for education talks from Chiesi, outside the submitted work.

\section{References}

1 Dodd JW, Getov SV, Jones PW. Cognitive function in COPD. Eur Respir J 2010; 35: 913-922.

2 Dodd JW, Charlton RA, van den Broek MD, et al. Cognitive dysfunction in patients hospitalized with acute exacerbation of COPD. Chest 2013; 144: 119-127.

3 Schou L, Ostergaard B, Rasmussen LS, et al. Cognitive dysfunction in patients with chronic obstructive pulmonary disease-a systematic review. Respir Med 2012; 106: 1071-1081.

4 Grant I, Heaton RK, McSweeny AJ, et al. Neuropsychologic findings in hypoxemic chronic obstructive pulmonary disease. Arch Intern Med 1982; 142: 1470-1476.

5 Sherman DS, Mauser J, Nuno M, et al. The efficacy of cognitive intervention in mild cognitive impairment (mci): a meta-analysis of outcomes on neuropsychological measures. Neuropsychol Rev 2018; 27: 440-484.

6 Addenbrookes Cognitive Examination (ACE)-III. http://dementia.ie/images/uploads/site-images/ACE-III_Administration_ (UK).pdf

7 Li J, Huang Y, Fei G-H. The evaluation of cognitive impairment and relevant factors in patients with chronic obstructive pulmonary disease. Respiration 2013; 85: 98-105.

$8 \mathrm{Li} \mathrm{J}, \mathrm{Fei} \mathrm{G}-\mathrm{H}$. The unique alterations of hippocampus and cognitive impairment in chronic obstructive pulmonary disease. Respir Res 2013; 14: 140.

9 Grant I, Prigatano GP, Heaton RK, et al. Progressive neuropsychological impairment and hypoxemia. Relationship in chronic obstructive pulmonary disease. Arch Gen Psychiatry 1987; 44: 999-1006.

10 Hung WW, Wisnivesky JP, Siu AL, et al. Cognitive decline among patients with chronic obstructive pulmonary disease. Am J Respir Crit Care Med 2009; 180: 134-137.

11 Thakur N, Blanc PD, Julian LJ, et al. COPD and cognitive impairment: the role of hypoxemia and oxygen therapy. Int J Chron Obstruct Pulmon Dis 2010; 5: 263-269.

12 Antonelli-Incalzi R, Gemma A, Marra C, et al. Chronic obstructive pulmonary disease: an original model of cognitive decline. Am Rev Respir Dis 1993; 148: 418-424.

13 Kupfer DJ, Regier DA, Narrow WE, et al. Diagnostic and Statistical Manual of Mental Disorders. 5th Edn. Washington, American Psychiatric Association, 2013.

14 Alzheimer's Disease International. Dementia Statistics. www.alz.co.uk/research/statistics Date last accessed: August $5,2018$.

15 Tombaugh TN, McIntyre NJ. The Mini-Mental State Examination: a comprehensive review. J Am Geriatr Soc 1992; 40: 922-935.

16 Pendlebury ST, Cuthbertson FC, Welch SJ V, et al. Underestimation of cognitive impairment by Mini-Mental State Examination versus the Montreal Cognitive Assessment in patients with transient ischemic attack and stroke: a population-based study. Stroke 2010; 41: 1290-1293.

17 Faustman WO, Moses JA, Csernansky JG. Limitations of the Mini-Mental State Examination in predicting neuropsychological functioning in a psychiatric sample. Acta Psychiatr Scand 1990; 81: 126-131.

18 Lezak M, Howieson D, Bigler D, et al. Neuropsychological Assessment. 5th Edn. USA, Oxford University Press, 2012.

19 Villeneuve S, Pepin V, Rahayel S, et al. Mild cognitive impairment in moderate to severe COPD: a preliminary study. Chest 2012; 142: 1516-1523.

20 Cleutjens FA, Janssen DJ, Gijsen C, et al. Cognitive impairment in patients with COPD: a review. Tijdschr Gerontol Geriatr 2014; 45: 1-9.

21 Ortapamuk H, Naldoken S. Brain perfusion abnormalities in chronic obstructive pulmonary disease: comparison with cognitive impairment. Ann Nucl Med 2006; 20: 99-106.

22 Dal Negro RW, Bonadiman L, Tognella S, et al. Extent and prevalence of cognitive dysfunction in chronic obstructive pulmonary disease, chronic non-obstructive bronchitis, and in asymptomatic smokers, compared to normal reference values. Int J Chron Obstruct Pulmon Dis 2014; 9: 675-683.

23 Antonelli-Incalzi C, Marra C, Giordano A. Cognitive impairment in chronic obstructive pulmonary disease - a neuropsychological and SPECT study. J Neurol 2003; 250: 325-332.

24 Joubert S, Gour N, Guedj E, et al. Early-onset and late-onset Alzheimer's disease are associated with distinct patterns of memory impairment. Cortex 2016; 74: 217-232.

25 Kent BD, Mitchell PD, McNicholas WT. Hypoxemia in patients with COPD: cause, effects, and disease progression. Int J Chron Obs Pulmon Dis 2011; 6: 199-128.

26 Frohnhofen H, Heuer HC, Willschrei HP, et al. Dementia among older in-hospital patients with obstructive airway disease. Frequency and consequences. Z Gerontol Geriatr 2011; 44: 240-244. 